\title{
Service Oriented D2D Efficient Communication for Post-Disaster Management
}

\author{
Sami Abdellatif*, Okba Tibermacine ${ }^{\llbracket}$, Walid Bechkit", Abdelmalik Bachir ${ }^{\S}$

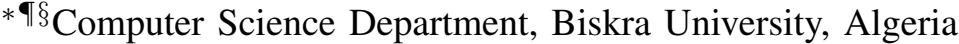 \\ "Univ Lyon, Inria, INSA Lyon, CITI, F-69621 Villeurbanne, France

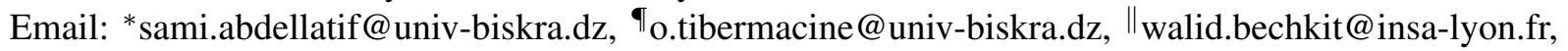 \\ §a.bachir@univ-biskra.dz
}

\begin{abstract}
In a post-disaster situation, the construction of replacement communication infrastructure is crucial for the success of rescue operations. LTE Device-to-Device Proximity Services and IoT are considered as key enabling technologies for the construction of such replacement networks. Existing techniques rely on smartphones as relay stations to build a replacement broadcast-based network that connects available devices. In many cases, the using of such networks require querying a given type of IoT devices (e.g. surveillance cameras, heart-rate monitors, temperature sensors) depending on network users and service requirements. In such scenarios, incorporating all relays in the broadcast is inefficient and may lead to poor network performance. In this paper, we propose constructing for each service type a sub-network of relay stations that ensure connectivity among IoT devices providing the same service type. The resulting sub-networks ensure an efficient and robust message dissemination, avoiding transmission redundancy, and resulting in higher energy savings as well as high coverage. These properties have been validated by implementing our solution in NS-3 by extending the LTE D2D ProSe module provided by NIST. Obtained results show significant improvements in terms of energy consumption, and packet delivery ratio.
\end{abstract}

Index Terms-Internet of Things; Services; Ad-Hoc; LTE ProSe; Post-Disaster

\section{INTRODUCTION}

Despite the great advancement in communication and networking technologies, there are still challenges to maintain main services operational when the infrastructure is fully or highly damaged. For example, in Nepal's earthquake in 2015, the entire telecommunication infrastructure was destroyed [1]. During that time companies such as Red Cross,Google and Facebook tried to provide tools to connect affected people and rescue teams. However, power failure and non-existence of last mile connectivity of communication networks, prevented nearly all connections between rescue teams, victims and survivors [2].

Solutions based on ad-hoc networks of smartphones such as [3] provide a viable alternative to traditional telecommunication infrastructure during these circumstances. However, their performance depends greatly on the wireless technology being used. For example, forming an ad-hoc network using short range wireless technologies (WiFi in most solutions) leads to poor communication due to constant disconnection and network instability.
Another major problem facing the rescue teams and aid workers during rescue operations is the lack of information on their environment and the state and location of victims. Although IoT devices are considered as a potential source of such information, where they provide vital services and information particularly in the cases of emergency and disaster situations, they rely on the traditional telecommunication infrastructure in order to be effective. This makes IoT devices and services unavailable in case the infrastructure is damaged. In addition, the previously mentioned ad-hoc based solutions lack the support for IoT devices and are more focused on smartphones and voice communication.

Recently, in its Releases 12 and 13, Third Generation Partnership Project (3GPP) proposed a wide range wireless communication technology based on LTE Device-to-Device (D2D) communication called Proximity Services (ProSe). ProSe allows direct device-to-device (D2D) broadcast communication between two or more User Equipment (UEs) that are in proximity of each other without passing through the LTE core network.

In this paper, we extend the one-hop LTE D2D ProSe broadcast to support multi-hop communication in an out-ofcoverage scenarios. We consider the case where rescuers need to query a certain type of devices and propose an Efficient D2D Broadcast algorithm based on requested service type. The main goal of this proposal is to further reduce the number of relays involved in message routing and optimize the traffic flow of each type of service. Figure 1 illustrates the network construction at phase I where we select a number of relays for general traffic, then phase II where we identify sub-networks for different types of services. In this example UE1, UE4, UE5, UE6, UE7 are selected to connect the entire network. The remaining smartphones become inactive during routing phase. For phase II, we assume that a rescuer is interested in monitoring victims heart states, then a request to smartwatches is going to be routed via most relay stations. Since, in this case UE6 does not cover the desired service, UE6 will remain inactive for this kind of traffic.

The main contributions of this work are as follow:

- An efficient multi-hop LTE D2D ProSe broadcasting mechanism based on a relay selection algorithm for outof-coverage situations.

- A multi path routing algorithm based on traffic type and requested IoT service. 


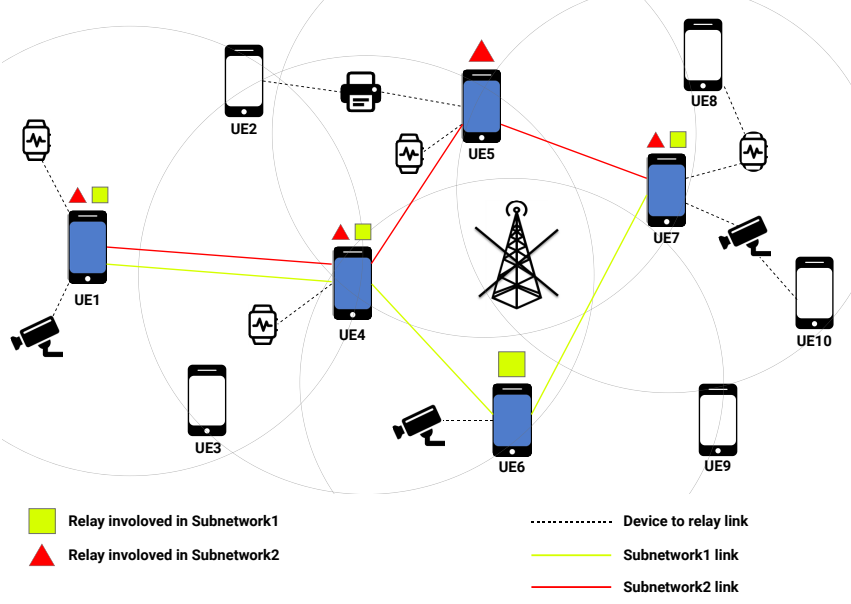

Fig. 1: Sub-networks identification for different types of services

- Implementing and testing of our proposed solution on top of a realistic LTE ProSe model provided by the National Institute of Standards and Technology (NIST) for NS3 simulator.

The remainder of this paper is organized as follows: Section II presents the related work and highlights the existing research gap. Section III and IV presents the network model and formulate the problem statement of this work. Section V describes the sub networks identification algorithm based on different types of services. Section VI presents the implementation and experimentation details. Section VII discusses the results obtained by the proposed solution using two different wireless interfaces WiFi and LTE ProSe. Section VIII concludes the paper and provides some future directions.

\section{RELATED WORK}

In literature, various IoT-based Disaster management systems have been proposed for monitoring and decision making during crisis. In [4]authors presented an emergency fire management system based on Web of things paradigm and a decision support tool to provide service features based on the context. In their proposal they emphasis on the importance of IoT data collection in rescue missions, but lake a way to connect remote IoT devices in such circumstances. To overcome this, [5] propose an energy aware data forwarding protocol that operates in two main phases : (i) a reasoning phase, to realize short terms objectives such as choosing the next hop based on remaining energy. (ii) A learning phase to assist in achieving long-term goals, such as improving network lifetime. The proposed network depends heavily on the existence of gateways, which makes IoT devices useless in the absence of such elements. In [6], both IoT devices and smartphones are involved in message forwarding. After receiving a message, an IoT device selects the best smartphone to relay data to, following two metrics: energy and connectivity degree. Similarly, [7], the authors presented a new ad-hoc network formation algorithm based on D2D communication. The algorithm starts by selecting relay agents that connect the affected area with the rest of the world. The selection of these relay agents is based on four criteria: (i) device residual energy, (ii) computational power, (iii) Channel Quality Index (CQI), and (iv) bandwidth availability. Although IoT devices are also capable of relaying messages, it can lead to depleting their small energy resources prematurely. In addition, their short range wireless interface, limits their ability to reach and help connecting isolated parts of the network.

In [8], a network of relay smartphones is formed to deliver rescue messages to central command stations. A proactive routing protocol is proposed for data forwarding, where routes are established by broadcasting advertisement messages sent from central command stations to network nodes. However, due to the range limitation of WiFi, Routes Establishment can fail, thus, communication can not be established between the command center and survivors in some cases. To overcome this, [9], focused on delivering health related information from victims to rescuers via LTE ProSe enabled Smartphones. First, these smartphones collect alert messages coming from wearable and e-health devices, then they broadcast received alert messages after embedding them in ProSe discovery messages. Although the proposed solution allows victims to reach rescuers instantly and over a long distance $(500 \mathrm{~m})$, it is only limited to one-hop transmission, and it requires the presence of traditional telecommunication infrastructure to enable D2D direct communications and to obtain a diagnosis or help instructions. In almost all proposals, authors have considered selecting relay nodes based on residual energy, processing power or link state quality. However, none of them took into consideration the type of traffic to be routed nor the significance of the IoT device to be connected. We can outline the limitations of existing solutions as (i) they usually rely on existing network infrastructure (mobile, broadband, Wi-Fi hotspots), that is prone to disruption during a disaster e.g. physical damage or as a result of overloading, (ii) IoT devices with limited resources participate in relaying data from one end to another (iii) Use a short range wireless interface in ad-hoc mode that results in network disconnection and poor performance.

To address these limitations and to provide rescuers a maximum access to IoT devices and services in an efficient way, we propose a multi hop ad-hoc network of LTE ProSe enabled smartphones. We introduce a selection algorithm to limit the number of relay smartphones involved in routing based on the type of service required. More details on the proposed solution can be found in Section V.

\section{NeTwork MOdEL AND ASSUMPTIONS}

In the rest of the paper, we distinguish between the following terms Device, Type, Station, Relay, and Node. A Device can be any small thing able to perform sensing or actuating operation and is generally very constrained in term of storage, processing and communication resources. We associate a Type to every device based on the services that it offers. A Station is a higher capacity communicating object, typically a smart phone equipped with LTE technology and held by rescuers, volunteers, or victims. In our case, we assume that it also 
implements D2D ProSe. A Node is a general term which is used to refer to a Device or a Station. A Relay is a Station involved in data forwarding from a Node to other Nodes.

We assume that the cellular network is totally damaged and out of service. We assume that rescuers, volunteers and victims have LTE D2D ProSe enabled smartphones and already installed our disaster relief application. The application allows users to (i) establish a mesh network with others directly via LTE D2D ProSe, (ii) discover, classify and collect data from surrounding IoT devices, (iii) exchange of geospatial information, help messages and other type of data. All devices are supposedly dispersed all over the effected area (e.g. vital signs monitoring devices, Smart-watches, Wearable devices, Wireless cameras, Temperature sensors, Presence sensors, etc.)

We assume that rescuers and volunteers are mobile, while victims are stationary waiting to be rescued. In addition, all stations are equipped with a USIM, providing all the preconfiguration and authorisation necessary for LTE D2D ProSe Communication specified in [10]

\section{Problem Formulation}

The main goal is to allow rescuers (or any other entity that uses the network) to query a group of devices or stations (certain type) simultaneously, in the most efficient way possible. In previous work we tried selecting an optimal set of relay nodes without taking into consideration the type of services covered by these relays. By neglecting this selection criterion, a number of relay nodes remain active during traffic routing unnecessarily, resulting in faster battery depletion.

Problem statement: Given the network graph $G(S, D, E)$ of Stations $S$ and IoT Devices $D$ connected via wireless links $E$. Let the ProSe cost, denoted $P\left\{n_{s}, n_{d}\right\}$ be the cost incurred by any request/response packet transmission from source node $n_{s}$ to destination node $n_{d}$ over multi-hop broadcast, s.t. $n_{s}$ and $n_{d}$ belongs to $S \cup D$. Every IoT Device is marked with a Type $T$ based on the service it offers. To minimise the ProSe cost for any communicating pairs $n_{s}$ and $n_{d}$, we build a sub-network for each type of service consisting of devices of the same Type $T$ and intermediary nodes that are necessary to connect these devices.

The Described problem is NP-complete, we prove this by showing that the above problem is similar to the minimum Connected Dominating Set (CDS), which is already known as NP-complete [11].However, considering two type of nodes (Station and IoT Devices) in the network, and constructing a sub network for each type of services, makes the problem more complex. In the following section, we discuss in detail the proposed heuristic to tackle the aforementioned problem.

\section{SERVICE ORIENTED EFFICIENT D2D BROADCAST}

We assume that we have $n$ Stations and $m$ services in the network. A station could be in three states: acting as Relay, Not acting as Relay, Undecided. We define the relaying state

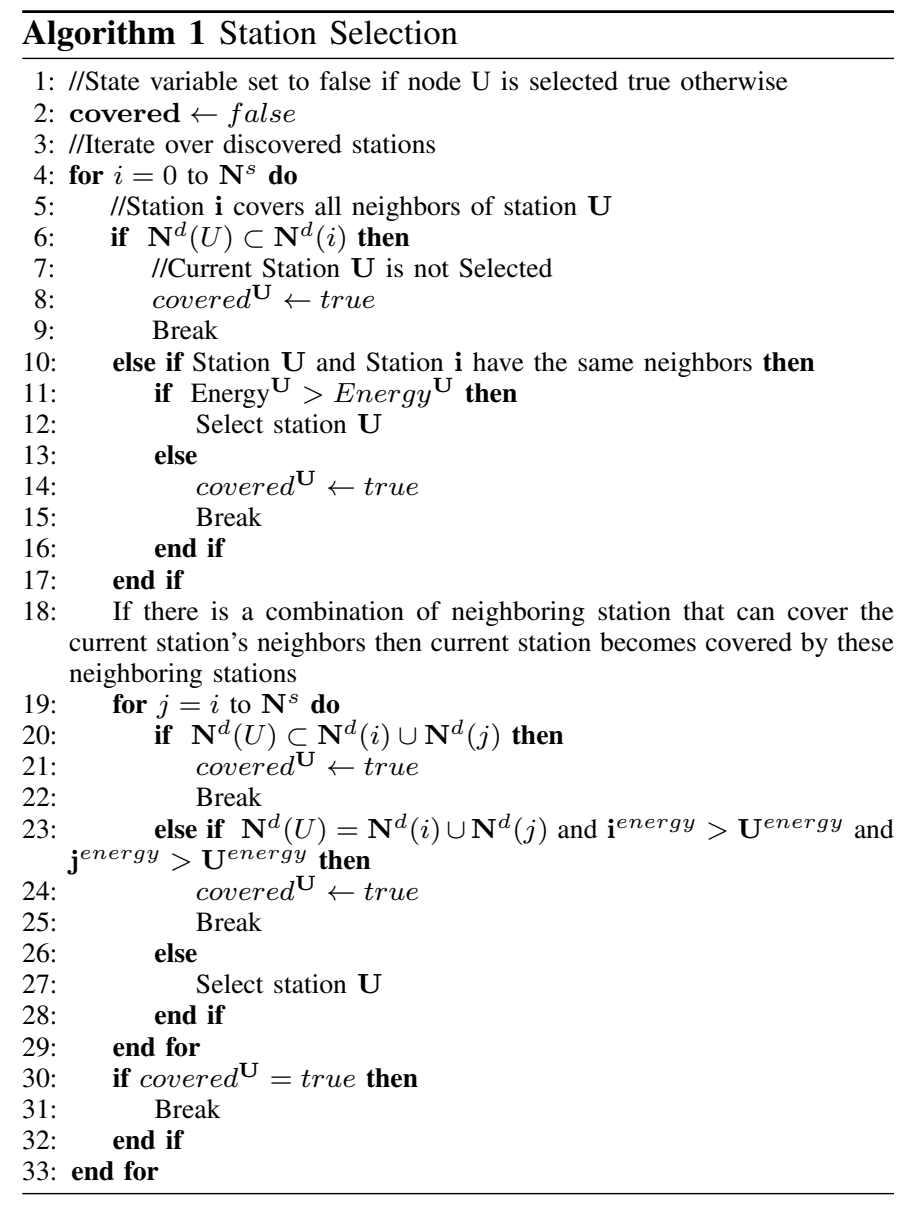

matrix $\mathbf{R}=r_{i j}$ for $i \in\{1, \ldots, n\}$ and $j \in\{1, \ldots, m\}$ where the state of each station $i$ is as follows :

$$
\begin{aligned}
& r_{i j}=-1 \text { initial state or undecided } \\
& r_{i j}=0 \text { if Station } i \text { is Not Relay for Service } j \\
& r_{i j}=1 \text { if Station } i \text { is Relay for Service } j
\end{aligned}
$$

Note that our solution is distributed, where every station keeps a list of covered services, and calculates its state for each service $j$. First we neglect the type of service and proceed with the relay selection, the result is a set of relays for general traffic where no service type is specified. Then, every relay station, calculates its states in regard to service $i$ and removes all other services from its list of neighbors, and runs the selection algorithm again with the updated neighbors list, if it is selected again as a relay then state $\{i\}=1$. Meaning that, the relay station must forward all traffic of service type $i$, else state $\{i\}=0$ and the station ignores any packet of service type $i$. As shown in figure 1 relay station UE6 remains inactive if the request is for a heart monitoring service. Algorithm 1 shows the periodic selection process for general traffic, whereas Algorithm 2 shows the service oriented relay selection process.

\section{A. Routing}

When receiving a request/response message, a relay node starts by checking the service type embedded in this messages. If the relay node is allowed to forward traffic of this type of 

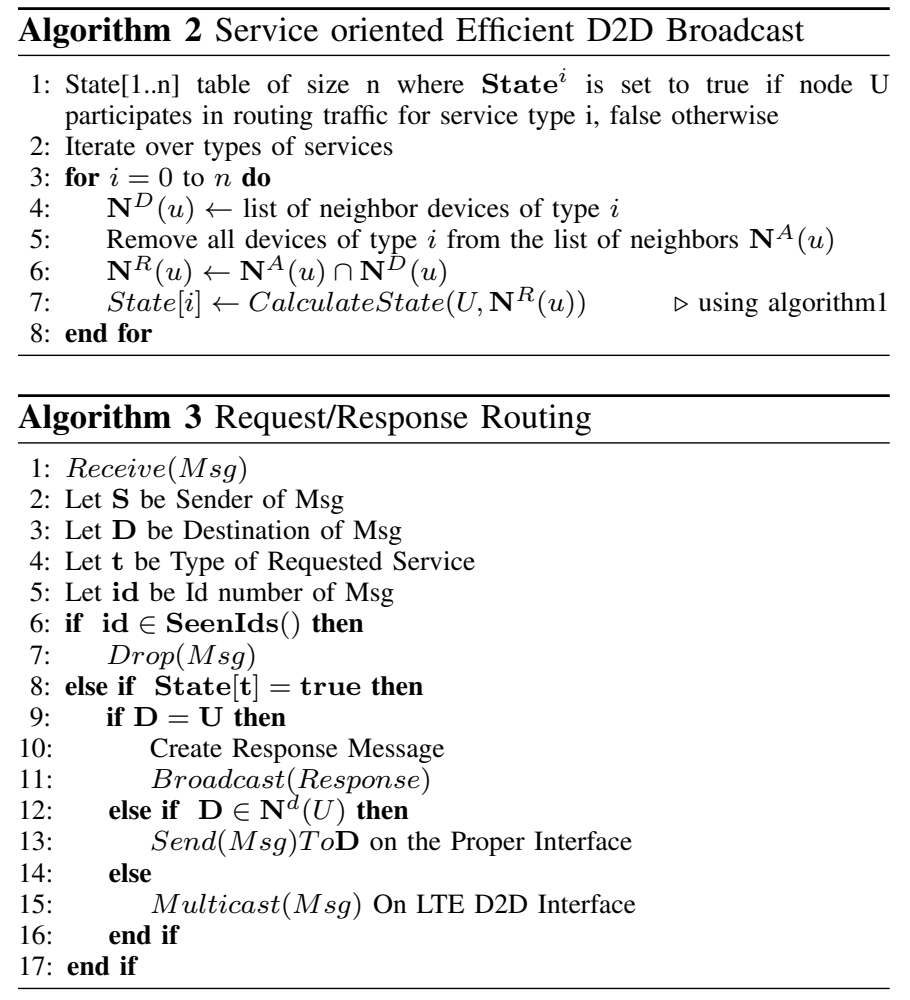

service state $\{i\}=1$ then it broadcast the message to it's destination, else it drops the message. Algorithms 3 shows the procedure of routing Requests and Responses messages based on service type.

\section{EVALUATION}

In this section we present an evaluation of the proposed solution through simulation in the discrete event network simulator ns-3 [12]. We built our simulation on top of the LTE ProSe module provided by NIST [13]. Nodes are deployed in an area of $500 \mathrm{~m} * 500 \mathrm{~m}$ randomly following a uniform distribution and divided into two groups, station and devices.

Stations are mobile, have a variety of wireless interfaces, namely LTE, 802.11 (Wi-Fi) and 802.15.4. Each station is equipped with an energy source of $10000 \mathrm{~J}$. The rest of the nodes (IoT devices) are stationary and randomly deployed across the simulation area. IoT devices only support 6LoWPAN over 802.15.4 and operate on a small size energy source of 100J. We use COST 231 Hata [14] propagation model across all our simulation scenarios.

In our simulation only stations perform the selection process. Data traffic consists of request/response packets and follows a Poisson process. For simulation purposes, we examine different network sizes. Table I summarizes the simulation configuration.

\section{A. Scenarios:}

In order to evaluate the performance gains provided by the Service oriented Efficient D2D Broadcast (SED2DB), in terms of energy consumption we test the new technique in different scenarios namely: (i) Different Network size: Here we start by varying the number of stations as well as the number of devices from 40 to 100 . Since our technique is service oriented, we vary the number of IoT devices from 50 to 200. The objective here is to test the effect of network size on the energy consumption.

(ii) Different network traffic: The type of traffic that needs to be routed throughout the network is important. We changed the destination of service requests from (station and devices) to devices only. In this scenario we assume that users are only interested in reaching IoT devices and their services. Also we changed the number of types of services that exists in the network.

(iii) Different Mobility Degree: The network topology usually has a huge effect on the network performance. To measure this we tested the proposed technique in different mobility speeds from low mobility $1 \mathrm{~m} / \mathrm{s}$ to high mobility $10 \mathrm{~m} / \mathrm{s}$.

TABLE I: Simulation Configuration

\begin{tabular}{ll} 
Parameters & Values \\
\hline \multicolumn{2}{c}{ Environment } \\
\hline Number of UEs & $20,30,40,50$ \\
Number of Devices & $50,100,150,200$ \\
Area size & $500 \mathrm{~m} \times 500 \mathrm{~m}$ \\
Stations Mobility & Random Walk \\
IoT Device Mobility & Stationary \\
Number of scenarios & 3 \\
Simulation period & $200 \mathrm{~s}$ \\
Total number of simulation per scenario & 100 \\
\hline & \\
\hline Number of resource pools & 1 \\
SideLink Bandwidth & 50 resource blocks \\
Resource block size & 4 \\
Carrier frequency & $700 \mathrm{MHz}$ \\
Discovery period & $0.32 \mathrm{~s}$ \\
PSCCH Length & 8 resource blocks \\
UE transmission power & $23.0 \mathrm{dBm}$ \\
Propagation loss model & Cost231 \\
\hline & \\
\hline Phy Wi-Fi & $802.11 \mathrm{n}$ \\
Tx Gain & $10 \mathrm{~dB}$ \\
Propagation Loss Model & Cost231 \\
Mac & Ad-hoc Wi-Fi Mac \\
\hline
\end{tabular}

\section{RESUlts AND ANALYSis}

In case of an emergency, preserving the lifetime of the replacement network is of a critical importance as well as ensuring a successful delivery of messages. Thus, we evaluate the performance of the proposed scheme based on these two important metrics. First, we generated a traffic (queries) destined for both stations and devices, as shown in figure 2 the energy consumption of our proposed solution (SED2DB) is less than the traditional D2D Broadcast (D2DB). However, the decrease is not significant (less than $10 \%$ in case of LTE ProSe, and lesser in case of $\mathrm{WiFi}$ ), this is due to all relay nodes remaining active during routing when a smartphone is the destination of the query being routed. To test this, we set the destination of queries to IoT devices only, and figure 3, shows a decrease in energy consumption that is more significant (around 20\% less energy consumption when LTE ProSe is used). 


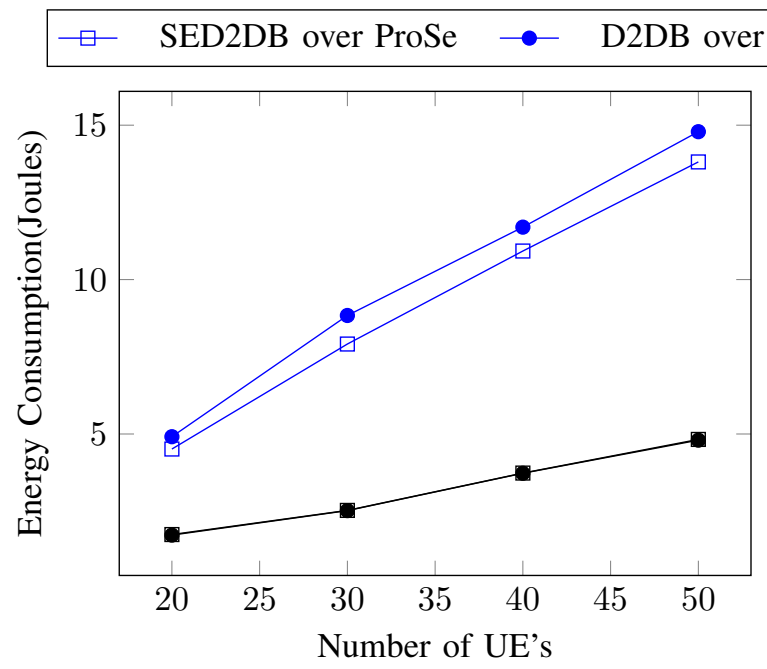

Fig. 2: Energy consumption in case queries are destined for both IoT devices and Smartphones

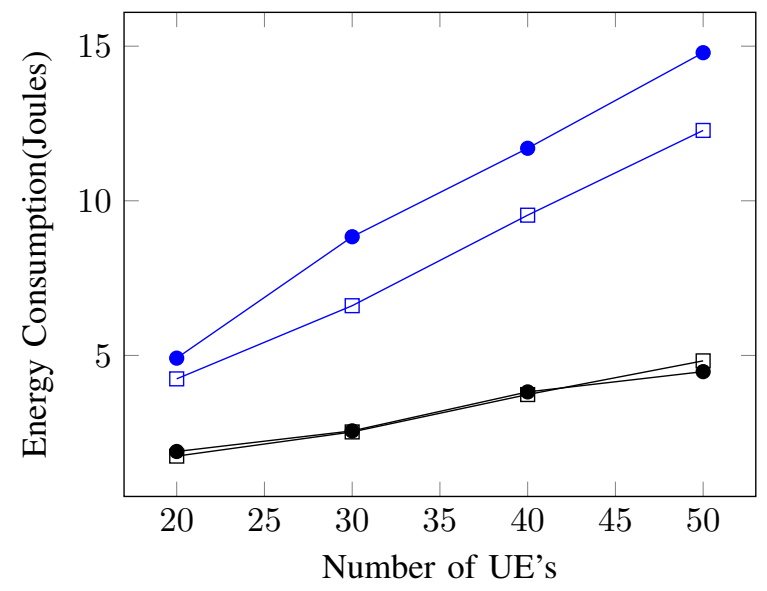

Fig. 3: Energy consumption in case queries are destined for IoT devices only

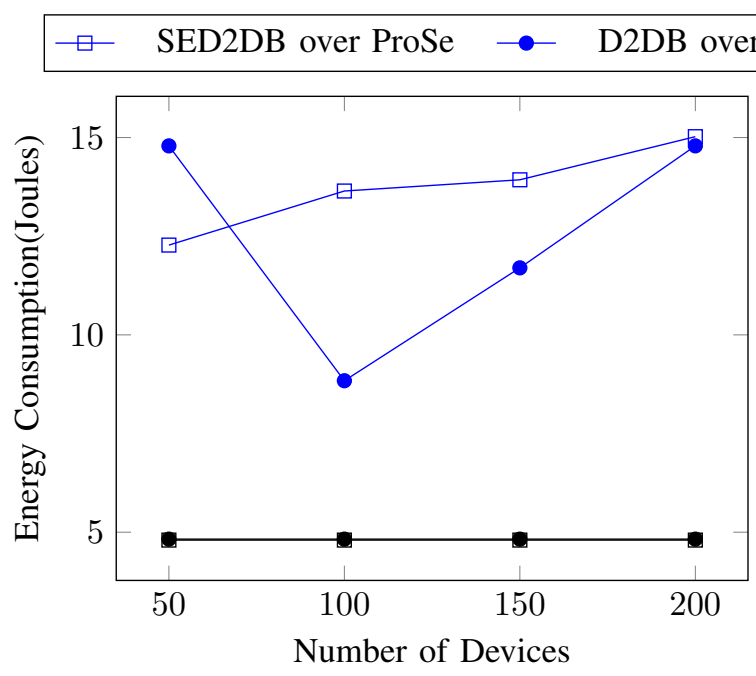

Fig. 4: Energy consumption vs Number of Devices

We then varied the mobility speed of nodes to simulate the behaviour of the network in low mobility as well as high mobility. As can be seen in figure 5 the higher the mobility the lesser the energy consumption for both simple (D2DB) and our solution (SED2DB), also we can notice that the performance of these two approaches is almost identical in high mobility cases. Figure 8 validates this where it shows the difference between both approaches, for example in low mobility $(1 \mathrm{~m} / \mathrm{s})$ our approach offer a $20 \%$ energy reduction, whereas in high mobility it becomes as low as $5 \%$, meaning that the proposed solution is more suited for low mobility networks. Figure 6 shows that the decrease of energy consumption of all the simulated approaches in high mobility cases comes at the expense of message delivery ratio, where it is clear that the higher the mobility is, the lower success chance of delivering

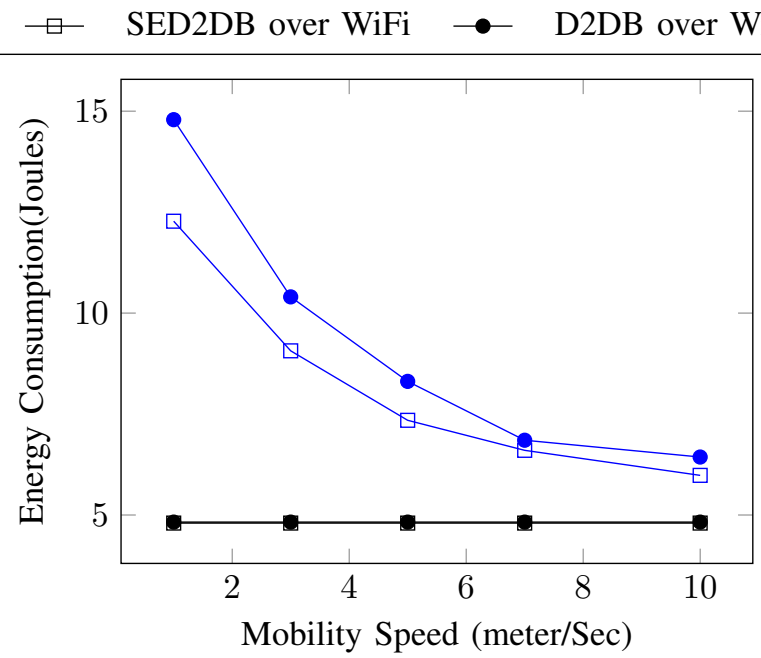

Fig. 5: Energy consumption per Mobility Speed a message to it's destination.

Since our solution is aimed for IoT devices and their type of services, we saw fit to test it in different types of services from few types to numerous types, and from figure 7 it can be seen that one of the big advantages of our proposes solution is that it scales well with the number of services in the network. As it is clear that the performance of (SED2DB) becomes better when the number of services increases.

\section{CONCLUSION}

We have proposed an efficient service oriented D2D broadcast on top of the ProSe direct communication for out of coverage situations. This solution is composed of two phases: in phase I, we construct a backbone of relay smartphones to forward general traffic and in phase II, we perform a dynamic 


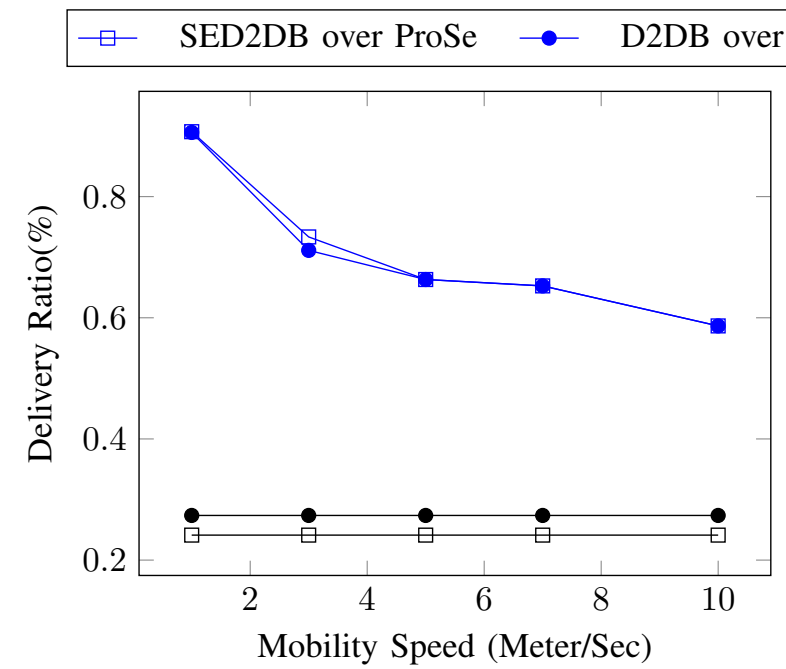

Fig. 6: Delivery Ratio per Mobility Speed

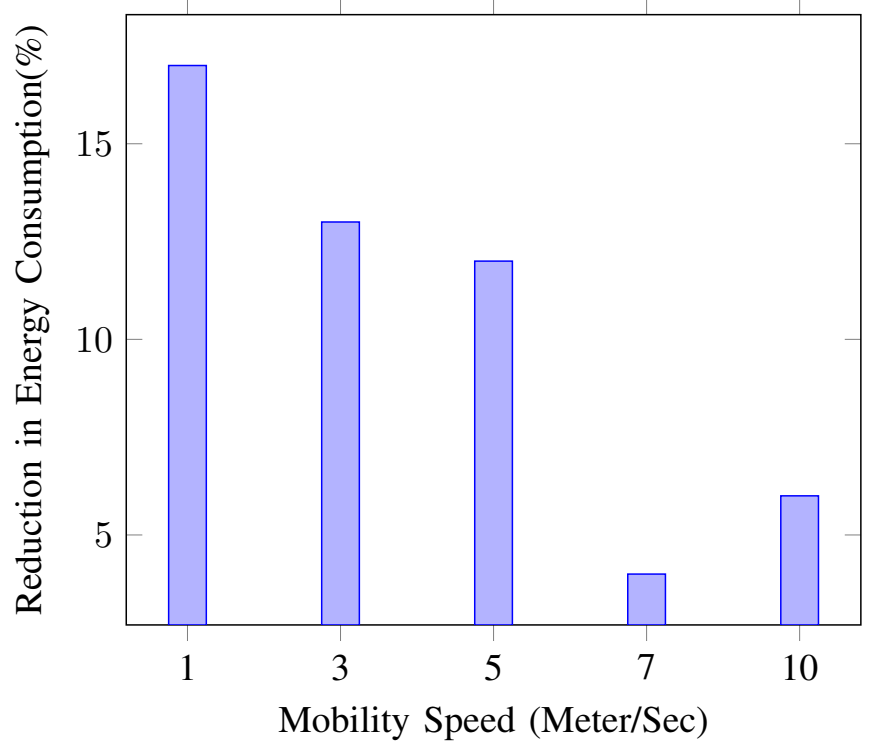

Fig. 8: Energy consumption reduction per Mobility Speed

relay selection on top of the constructed backbone and based on the type of required service and network traffic. We have shown that constructing for each service type a sub-network of relay stations yields significant performance gains, especially in preserving network lifetime. We have also shown that the proposed solution is best suited for low mobility networks, and requires additional improvements for high mobility situations.

\section{REFERENCES}

[1] H. Chen, Q. Xie, B. Feng, J. Liu, Y. Huang, and H. Chen, "Seismic performance to emergency centers, communication and hospital facilities subjected to nepal earthquakes, 2015," Journal of Earthquake Engineering, vol. 22, no. 9, pp. 1537-1568, 2018.

[2] Y. Segal, "Is there a solution to the problem of communications in natural disasters?."

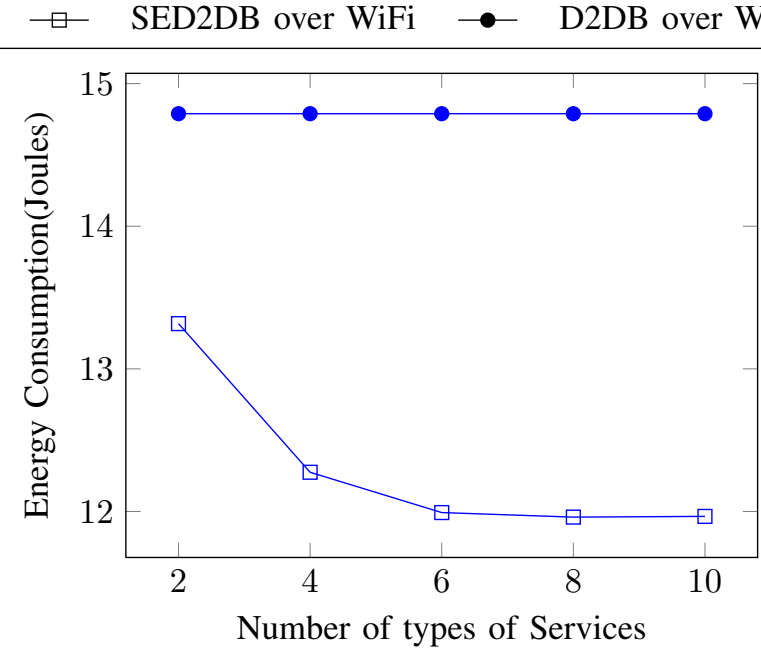

Fig. 7: Energy consumption per number of types of services

[3] Z. Lu, G. Cao, and T. La Porta, "Teamphone: Networking smartphones for disaster recovery," IEEE Transactions on Mobile Computing, vol. 16, no. 12 , pp. 3554-3567, 2017.

[4] Z. U. Shamszaman, S. S. Ara, I. Chong, and Y. K. Jeong, "Web-ofobjects (woo)-based context aware emergency fire management systems for the internet of things," Sensors, vol. 14, no. 2, pp. 2944-2966, 2014.

[5] F. Al-Turjman, "Cognitive routing protocol for disaster-inspired internet of things," Future Generation Computer Systems, vol. 92, pp. 11031115, 2019.

[6] M. A. Hossain and S. K. Ray, "A smartphone-assisted device-to-device communication for post-disaster recovery," in International Conference on Smart Grid Inspired Future Technologies, pp. 11-20, Springer, 2018.

[7] M. Kamruzzaman, N. I. Sarkar, J. Gutierrez, and S. K. Ray, "A study of iot-based post-disaster management," in 2017 International Conference on Information Networking (ICOIN), pp. 406-410, IEEE, 2017.

[8] D. B. Arbia, M. M. Alam, R. Attia, and E. B. Hamida, "Orace-net: A novel multi-hop body-to-body routing protocol for public safety networks," Peer-to-Peer Networking and Applications, vol. 10, no. 3, pp. 726-749, 2017.

[9] P. Bellavista, J. De Benedetto, C. R. De Rolt, L. Foschini, and R. Montanari, "Lte proximity discovery for supporting participatory mobile health communities," in Communications (ICC), 2017 IEEE International Conference on, pp. 1-6, IEEE, 2017.

[10] Universal mobile telecommunications system (UMTS), proximity-based services (ProSe); Stage 2, Apr 2016. Rev. 12.0.

[11] M. Chlebík and J. Chlebíková, "Approximation hardness of dominating set problems in bounded degree graphs," Information and Computation, vol. 206, no. 11, pp. 1264-1275, 2008.

[12] G. F. Riley and T. R. Henderson, "The ns-3 network simulator," in Modeling and tools for network simulation, pp. 15-34, Springer, 2010.

[13] R. Rouil, F. J. Cintrón, A. Ben Mosbah, and S. Gamboa, "Implementation and validation of an lte $\mathrm{d} 2 \mathrm{~d}$ model for ns-3," in Proceedings of the Workshop on ns-3, pp. 55-62, ACM, 2017.

[14] P. Lähdekorpi, T. Isotalo, K. Kylä-Liuhala, and J. Lempiäinen, "Replacing terrestrial umts coverage by hap in disaster scenarios," in 2010 European Wireless Conference (EW), pp. 14-19, IEEE, 2010. 